\title{
SIP for Call Control in the 3G IP-Based UMTS Core Network
}

\author{
Delphine Plasse \\ BT Adastral Park, UK \\ delphine.plasse@bt.com
}

\begin{abstract}
This paper present an example of call control using the Session Initiation Protocol (SIP) for multimedia call management in the "all IP" architecture for the core network of UMTS. The first part of this publication gives an overview of the 3GPP IP-based UMTS core network. The second part introduces an interworking scenario between the UMTS core network and the SIP-enabled public Internet. The current main issues are highlighted.
\end{abstract}

\section{Introduction}

The $3^{\text {rd }}$ Generation Partnership Program group (3GPP) [1] is in the process of specifying the core network architecture of UMTS phase 2 also known as Release 2000 (R00). A number of options have been proposed for the R00 architecture, one of which known as the UMTS "all-IP" architecture:

The "all IP" architecture is evolving from the GSM/GPRS architecture-based UMTS phase 1 (R99). It is intended to use IP components within the core network, with evolved GPRS elements supporting data, multimedia and voice over IP (VoIP) mechanisms for the delivery of, for example, speech and traditional circuit-switched type of features.

The "all IP" architecture is required to provide interworking solutions between UMTS and $2^{\text {nd }}$ Generation mobile networks, the legacy PSTN/ISDN networks and external IP-based SIP/ H323-enabled networks. It is also required to support standardised services, Camel Service Switching Functions, call control and Media Gateway interrogation. The investigation of solutions for multimedia call management is an important issue in the 3GPP group. A possible solution is the application of IP-based signalling for call control in the "all-IP" architecture.

The first part of this paper introduces the UMTS R00 architecture. The second part will present a solution employing the SIP protocol [2], for multimedia call set-up and termination between UMTS/GPRS network and external multimedia IP-based networks. 


\section{The UMTS IP-Based Architecture [3]}

The UMTS "all-IP" architecture is based on the evolution of the GPRS core network standard, which will fully support advanced Voice over IP, data and multimedia applications.

Figure 1 shows a simplified overview of the Reference Architecture for Release 2000. The final approval of this architecture will depend on the resolution of a certain number of issues, which include the relationship between different call control models. The following section is describing the various functional elements:

CSCF: the Call State Control Function. It performs call control, service-switching functions, address translation functions and vocoder negotiation functions.

HSS: the Home Subscriber Server. It is the master database for a given user. It is responsible for keeping a master list of features and services (either directly or via servers) associated with a user, and for tracking of location and means of access for its users. It provides user profile information, either directly or via servers. It is a superset of the HLR functionality, since it also communicates via new IP-based interfaces.

MGCF: the Media Gateway Control Function. It is the point of interface for signalling between IP-based packet switched networks i.e. GPRS, and circuit switched networks. The MGCF communicates with the CSCF. It selects the CSCF depending on the routing number for incoming calls from legacy networks and

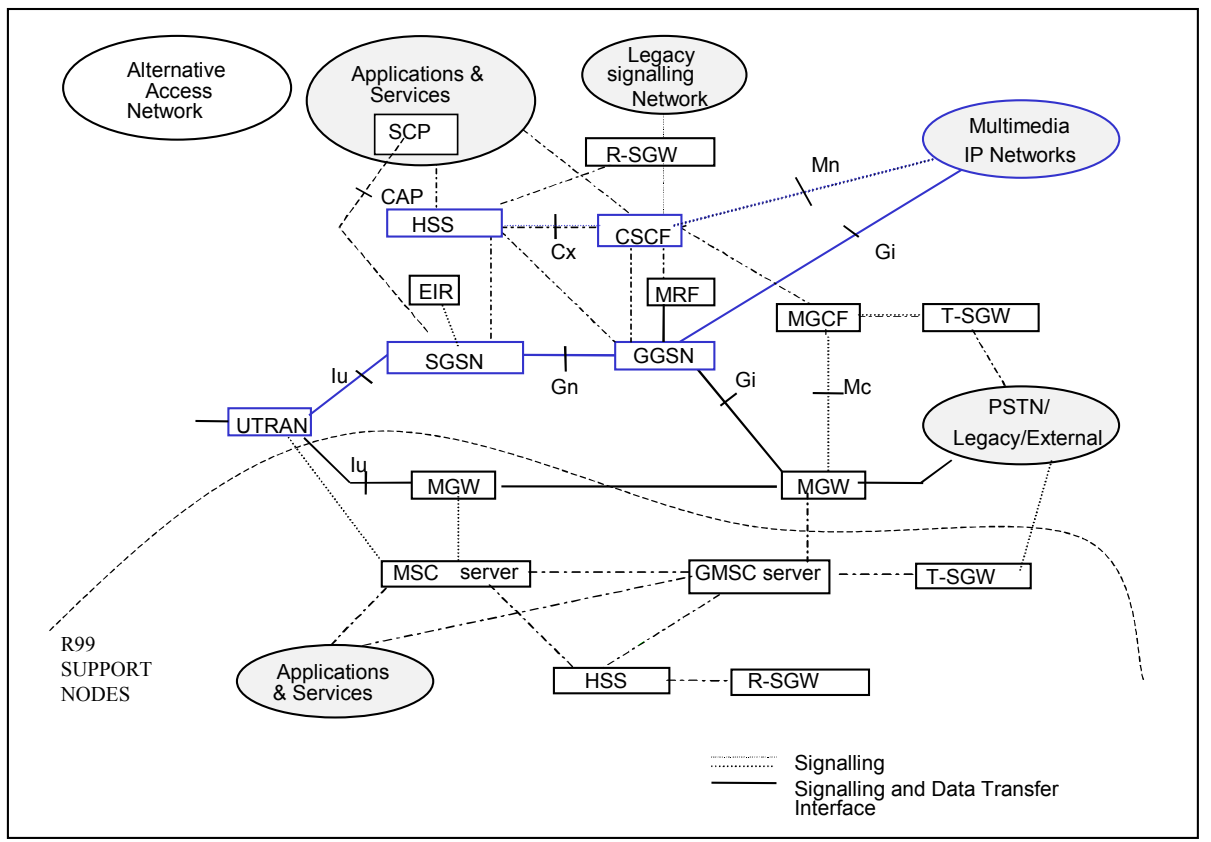

Fig. 1. Reference Architecture for Release 2000 [1] 
performs protocol conversion between the Legacy (e.g. ISUP, R1/R2 etc.) and the R00 network call control protocols. Out of band information is assumed to be received in MGCF and may be forwarded to $\mathrm{CSCF} / \mathrm{MGW}$.

$M G W$ : the Media Gateway function. This component is the PSTN/PLMN transport termination point for a defined network, and interfaces UTRAN with the core network over the Iu interface. A MGW may terminate bearer channels from a circuit switched network and may terminate media streams from a packet network (e.g., RTP streams in an IP network). It may support media conversion, bearer control and payload processing (e.g. codecs, echo canceller, conference bridge) for support of different Iu options for circuit switched services: AAL2/ATM based as well as RTP/UDP/IP based. The MGW interacts with the MGCF, the MSC server and GMSC server for resource control.

$T$-SGW: the Transport Signalling Gateway Function. This R00 network component is the PSTN/PLMN termination point for a defined network. It maps call related signalling from/to PSTN/PLMN on an IP bearer and sends it to/from the MGCF. It also needs to provide PSTN/PLMN to IP address mapping.

$R-S G W$ : The Roaming Signalling Gateway function. It supports roaming to/from $2^{\text {nd }}$ Generation/R99 circuit Switched to/from R00 UMTS GPRS domain. It provides signalling conversion between legacy SS7 based signalling and the IP based signalling to support roaming.

MSC server: The MSC server caters for R99 roaming users on the R00 network. It comprises the call control and mobility control parts of a GSM/UMTS R99 MSC, in an IP-based network. The MSC server would cater for VLR and control parts of MSC \& GMSC.

GMSC server: the Gateway MSC Server. The GMSC comprises mainly the call control and mobility control parts of a GSM/UMTS GMSC.

\section{Session / Call Control}

In the UMTS IP-based core network, it is the role of the CSCF to perform session / call control. Although the CSCF functions are still being studied, the main CSCF functionality have been highlighted:

Call registration, routing of incoming calls, call set-up / termination, call states and event management, call event reports for billing \& auditing, support of multi-party services, as well as address handling \& cache management, communication with the HSS, MRF and MGW, and provision of service trigger mechanisms.

The SIP protocol is currently being studied as a possible protocol for multimedia call management in the "all - IP " UMTS architecture. SIP has been designed for the initiation and termination of any type of sessions across the Internet [4]:

Determination of the communicating end system(s), determination of the media parameters to be used, determination of the willingness of the called party to engage in communication, call transfer and termination of calls, and set-up of the call parameters at both called and calling party.

SIP signalling has many similarities with HTTP in terms of header field structure, encoding rules and authentication mechanisms, and SMTP in terms of addressing scheme, address resolution or server location mechanisms. 
As shown in the following section, the SIP architecture enables a large number of call management functions [5]:

Application registration / authorisation with SIP REGISTER messages and events management messages such as the 100 message series. SIP supports multi-party services and address handling with its name to multiple address resolution schemes. Cache management and communication with location databases and gateways cater for the invocation of location-based services, while INVITE messages trigger all type of call services [6].

\section{Using SIP to Support Call Control}

Figures 2 and 3 shows an example of the usage of the SIP protocol for call control. The example is a successful UMTS originated call to a public Internet SIP-enabled terminal B. This call control scenario shows the UMTS terminal A registration with the CSCF / SIP server, then the scenario shows the initiation / termination of the multimedia session between a UMTS terminal and an Internet SIP-based terminal. A network resource reservation phase needs to take place in order to cater for real time traffic requirements (in our example, a VoIP session).

Certain assumptions have been made: the mobile user A is in his/her home PLMN. Terminal A has already discovered which CSCF to register with. User B is already registered with the network. SIP servers may be acting in proxy mode should they not be able to handle requests themselves.

The Registration phase either validates or invalidates a SIP client for user services provided by the SIP server:

\section{UMTS/ GPRS}

\section{Public Internet}

\section{CSCF}

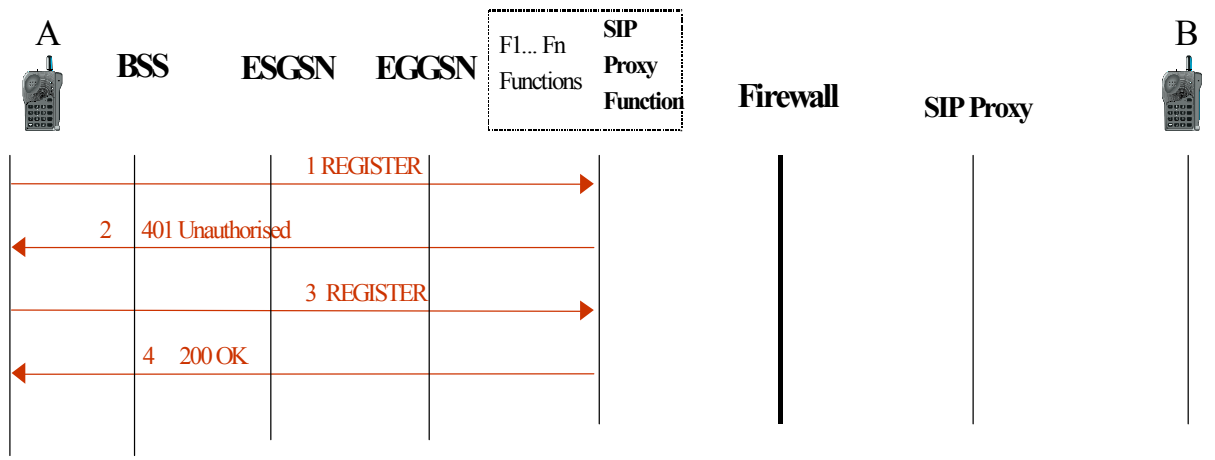

Fig. 2. Non-roaming user A registers with its home CSCF. 
1. User A sends a SIP REGISTER message to its home CSCF / SIP (proxy) server.

2. The SIP proxy server provides an authorization challenge to user A (the SIP digest format is described in RFC 2543).

3. User A enters his/her valid user Identifier and password, encrypted according to the challenge issued by the SIP server and sends the response to the SIP server.

4. The SIP proxy validates the user's credentials. It registers the user in its location database (which could be the user's UMTS HSS) and returns a $200 \mathrm{OK}$ response to user A.

In the next phase, user A completes a call to user B. The successful call shows the initial signalling and the exchange of media information in the form of SDP payloads, the establishment of the media session, then finally the termination of the call:

\section{UMTS / GPRS}

\section{CSCF}

\section{Public Internet}

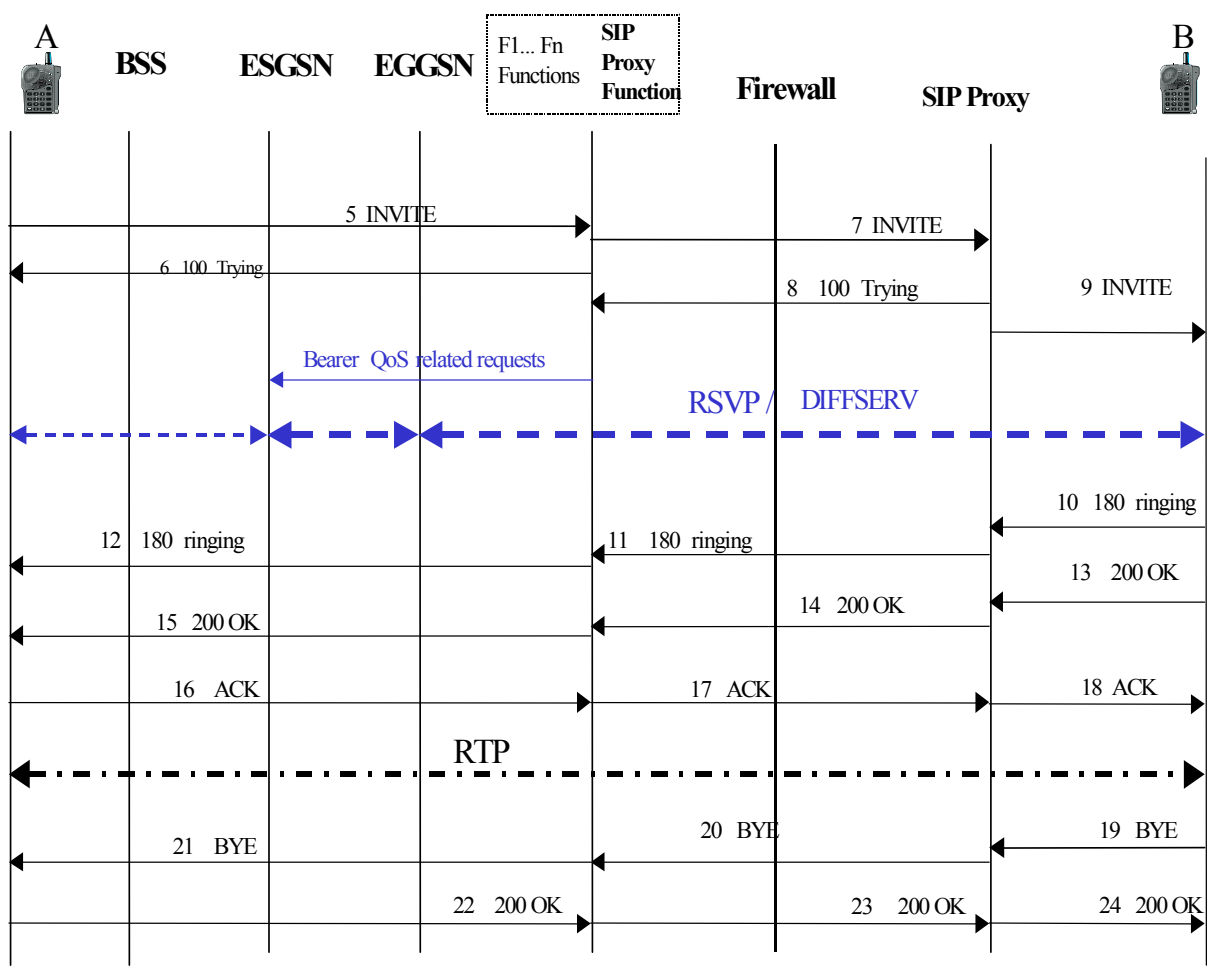

Fig. 3. UMTS originated VoIP call to a public Internet SIP enabled terminal B

5. User A's client sends an INVITE message to its UMTS CSCF.

6. A's CSCF accepts the credentials and forwards the INVITE message to B's SIP proxy server. A 's CSCF is assumed to have been authenticated by B 's proxy. Both CSCF and proxy have inserted their Record-Route header into the INVITE message to ensure that it is present in all subsequent message exchanges. 
7-12. "Trying" and "Ringing" event messages are sent to communicating parties, i.e. user A "s user agent client, and both CSCF and SIP proxies. Since SIP supports multi party call, the initial INVITE message may contains more than one "via" message header and may have been forked. Therefore user B client will insert a tag in message 10.

13-18. User B, its home proxy and the caller's CSCF accept the session invitation and receive communicating party acknowledgment messages. RTP streams are established between user A and user B and data transfer takes place.

19-24. User B hangs up with user A and the VoIP session terminates with a BYE message initiated by user B to its proxy server, and cascaded to user A via its $\mathrm{CSCF}$. In return, user A confirms the session termination.

\section{Open Issues}

We assumed that users were not roaming. But what happens when a user registers from a visited network? Should the registration /session control logic be executed in the visited network or in the home network?

Once the CSCF decides to admit the call, network resources need to be reserved in order to support the call traffic requirements (Voice traffic). A priori, the network resources ought to be established before end users answer the call. A dialog between the CSCF and the calling terminal, or perhaps, with a GPRS resource manager node (as shown in Figure 3) needs to take place in order to trigger the GPRS bearer resource allocation. It is also necessary for resource managers within the external IPbased network and within the UMTS network to interwork in order to perform end to end resource management. Similarly, once the session is finished (BYE messages are sent), they need to be freed up. How these mechanisms could be handled are open issues. SIP session signalling does not provide resource reservation, but it can convey the appropriate information to the appropriate network resource managers.

\section{Conclusion}

This paper presented an overview of the UMTS R00 architecture. It also showed that SIP signalling, message interactions and architecture offer sets of functionality for call control services that can be used in the "all-IP" UMTS architecture. As a simple example, a successful case of a UMTS originated VoIP session to a public Internet SIP- enabled terminal B was presented. A number of open issues have also been introduced. As work progresses in this area other issues will emerge. Future work will try and answer them. It will tackle mobile originated, mobile terminated calls for roaming users, in both successful and failure cases. 


\section{References}

1. Www.3gpp.org

2. "SIP: Session Initiation Protocol", M. Handley, H. Schulzrinne, E. Schooler, and J. Rosenberg, RFC 2543, March 1999.

3. "Architecture Principle for Release 2000", TR 23.821 v 0.1.0, 3GPP SA.

4. "www.ietf.org

5. H. Schulzrinne and al, "SIP Telephony Call Flow Examples", Internet draft, March 00, work in progress.

6. "H. Schulzinne et al., "Implementing Intelligent Network Services with the Session Initiation Protocol", Tech-Report Number CUCS-002-99, Columbia University.

\section{Short Glossary}

E-GPRS: Enhanced General Packet Radio Service.

HTTP: Hyper Text Transfer Protocol.

IP: Internet Protocol.

PLMN: Public Lan Mobile Network

SIP: Session Initiation Protocol.

SMTP: Simple Message Transfer Protocol.

RTP: Real Time Protocol.

UMTS: Universal Mobile Telecommunication Services. 